\title{
A Joint Distribution of Extreme Value for a Renewal Risk Model with Interest Force
}

\author{
Guojin Xu and Huibing Hao \\ Department of Mathematics, Hubei Engineering University, Hubei Xiao gan, 432000 \\ xgxysxx@163.com, haohuibing@163.com
}

Keywords: Interest force; Renewal risk model; The joint distribution of the minimum surplus and maximum surplus before the ruin.

Abstract. In this paper, we consider a renewal risk model with interest force, derive the joint distribution of the minimum surplus and maximum surplus before the ruin, and the integral equation of the joint distribution is also obtained.

\section{Introduction}

In recent years, the classic risk model has received a remarkable amount of attention and there have been many generalizations. Sundt and Teugels $(1995,1997)$ considered a compound Poisson model with a constant interest force, and the upper and lower bounds for the ruin probability and the integral equation of the ruin probability were obtained by using renewal techniques. Yang and Zhang (2001a, 2001b, 2001c) used the techniques of Sundt and Teugels (1995), some related problems were obtained. Yang (1998) considered a discrete time risk model with a constant interest force, and both Lundberg-type inequality and non-exponential upper bounds for ruin probabilities were obtained by using martingale inequalities. Renewal risk model with interest force as a generalization of the classic risk model was considered in $\mathrm{Wu}$ and $\mathrm{Du}$ (2002) by using discrete method. Lin and Wang (2005) adopted a different discrete techniques, derived the distribution of surplus immediately before ruin and that of deficit at ruin, further the integral equations of these distributions were obtained.

In this paper, we consider renewal risk model with interest force. By using the techniques of Lin and Wang (2005), the joint distribution of the minimum surplus and maximum surplus before the ruin is derived, and the integral equation of the joint distribution is also obtained.

\section{Definition of the Model}

Let $(\Omega, F, P)$ be a complete probability space. We consider the renewal risk model with interest force. Suppose $^{S(t)}$ denote the amount of claim in the time interval ${ }^{(0, t]}$, i.e. $S(t)=\sum_{i=1}^{N(t)} X_{i}$, where $\left\{X_{i}, i \geq 1\right\}$ is independent and identically distributed (i.i.d.) random variables with common distribution function $F(x)$, denotes the amount of the $i$ th claim. The counting process $\{N(t), t \geq 0\}$ denotes the number of claims up to time ${ }^{t}$

and is defined as

$$
N(t)=\max \left\{k: W_{1}+W_{2}+\cdots+W_{k} \leq t\right\} \text {, where the inter-claim times }\left\{W_{i}, i \geq 1\right\} \text { are assumed to be }
$$

i.i.d. random variables with common distribution function $K(w)$. Further, we assume the sequences $\left\{W_{i}, i \geq 1\right\}$ and $\left\{X_{i}, i \geq 1\right\}$ are independent, and that $c E\left(W_{1}\right)>E\left(X_{1}\right)$, providing a positive safety loading factor.

Let $U_{\delta}(t)$ denotes the insurance company's surplus at time ${ }^{t}$. From the above assumption, it follows that

$$
d U_{\delta}(t)=c d t+U_{\delta}(t) \delta d t-d S(t)
$$


From Sundt and Teugels (1995) and (1), we know that

$$
U_{\delta}(t)=u e^{\delta t}+c \bar{S}_{\bar{t} \mid}-\int_{0}^{t} e^{\delta(t-v)} d S(v)
$$

where

$$
\bar{S}_{\bar{t} \mid}=\int_{0}^{t} e^{\delta v} d v=\left\{\begin{array}{cll}
t & \text { if } & \delta=0 \\
\frac{e^{\delta t}-1}{\delta} & \text { if } & \delta>0
\end{array}\right.
$$

$u>0$ is initial surplus of insurance company, $c>0$ is the premium income of unite time, $\delta$ is constant interest force.

Definition1. If $T=\inf \left\{t>0: U_{\delta}(t)<0\right\}(T=\infty$ if the set is empty), $T$ is the ruin time. Obviously, it's a stopping time.

Definition2. Let $\Psi_{\delta}(u)$ denote the ultimate ruin probability with initial reserve $u$, That is

$$
\Psi_{\delta}(u)=P\left\{\bigcup_{t \geq 0}\left(U_{\delta}(t)<0 \mid U_{\delta}(0)=u\right\}\right.
$$

\section{Main Results}

Let $T_{n}$ denote the time of the $n$th claim happening, i.e. $T_{n}=\sum_{i=1}^{n} W_{i}$. By (2) we have

$$
U_{\delta}(t)=u e^{\delta t}+c \frac{e^{\delta t}-1}{\delta}-\sum_{i=1}^{n} X_{i} e^{\delta\left(t-T_{i}\right)}
$$

when $t=T_{n}$, we have

$$
\begin{aligned}
U_{\delta}\left(T_{n}\right)=u e^{\delta T_{n}} & +c \frac{e^{\delta T_{n}}-1}{\delta}-\sum_{i=1}^{n} X_{i} e^{\delta\left(T_{n}-T_{i}\right)} \\
& =u e^{\delta T_{n}}-\sum_{i=1}^{n}\left[X_{i}-c \frac{e^{\delta W_{i}}-1}{\delta}\right] e^{\delta\left(T_{n}-T_{i}\right)} \\
& =u e^{\delta \sum_{i=1}^{n} W_{i}}-\sum_{i=1}^{n} Y_{i} e^{\delta\left(T_{n}-T_{i}\right)} \\
& =u e^{\delta \sum_{i=1}^{n} W_{i}}-\sum_{i=1}^{n} Y_{i} e^{\delta \sum_{j=i+1}^{n} W_{j}}
\end{aligned}
$$

where

$$
Y_{i}=X_{i}-c \frac{e^{\delta W_{i}-1}}{\delta}, i \geq 1
$$

Obviously $\left\{\left(Y_{i}, W_{i}\right), i \geq 1\right\}$ are independent and have the same distribution $G(y, w)$,

$$
\begin{aligned}
G(y, w)=P\left\{Y_{1}\right. & \left.=X_{1}-c \frac{e^{\delta W_{1}}-1}{\delta} \leq y, W_{1} \leq w\right\} \\
& =\int_{0}^{w} P\left\{X_{1}-c \bar{S}_{\bar{t} \mid} \leq y\right\} d K(t) \\
& =\int_{0}^{w} F\left(y+c \bar{S}_{\bar{t} \mid}\right) d K(t)
\end{aligned}
$$

For $a>0, b>0$, let $H(u, a, b)$

$$
H(u, a, b)=P\left\{\inf _{0 \leq t<T} U_{\delta}(t) \geq b, \sup _{0 \leq t<T} U_{\delta}(t) \leq a, \quad T<\infty \mid U_{\delta}(0)=u\right\}
$$

be the joint distribution function of the minimum surplus and maximum surplus before the ruin with the initial reserve $u$.

Theorem 1. Let $H(u, a, b)$ be defined as (3), then we have

(1) when $u>a$ or $u<b$, then $H(u, a, b)=0$ 
(2) when $b \leq u \leq a$, then $H(u, a, b)=\sum_{n=1}^{\infty} h_{n}(u, a, b)$,

Where

$$
\begin{gathered}
h_{1}(u, a, b)=\int_{0}^{\infty} \bar{F}\left(u e^{\delta t}+c \bar{S}_{\bar{t} \mid}\right) d K(t) \\
h_{n}(u, a, b)=\int_{0}^{\infty} \int_{u e^{\delta t}+c \bar{S}_{\bar{T} \mid}-a}^{u \delta t} h_{n-1}\left(u e^{\delta t}+c \bar{S}_{\bar{t} \mid}-y, a, b\right) d F(y) d K(t)
\end{gathered}
$$

Proof (1) when $u>a$ or $u<b$, according to definition (3), obviously $H(u, a, b)=0$.

(2) when $b \leq u \leq a$, according to definition (3), we have

$$
\begin{gathered}
H(u, a, b)=P\left\{\inf _{0 \leq t<T} U_{\delta}(t) \geq b \sup _{0 \leq t<T} U_{\delta}(t) \leq a, T<\infty\right\} \\
=\sum_{n=1}^{\infty} P\left\{\inf _{0 \leq t<T} U_{\delta}(t) \geq b \sup _{0 \leq t<T} U_{\delta}(t) \leq a, T=T_{n}\right\} \\
=\sum_{n=1}^{\infty} P\left\{b \leq U_{\delta}\left(T_{1}\right) \leq a, b \leq U_{\delta}\left(T_{2}\right) \leq a\right. \\
\left.\quad \cdots, b \leq U_{\delta}\left(T_{n-1}\right) \leq a, U_{\delta}\left(T_{n}\right)<0\right\} \\
=\sum_{n=1}^{\infty} h_{n}(u, a, b)
\end{gathered}
$$

where

$$
h_{n}(u, a, b)=P\left\{b \leq U_{\delta}\left(T_{1}\right) \leq a, b \leq U_{\delta}\left(T_{2}\right) \leq a, \cdots, b \leq U_{\delta}\left(T_{n-1}\right) \leq a, U_{\delta}\left(T_{n}\right)<0\right\}
$$

According to the definition, we have

$$
\begin{aligned}
h_{1}(u, a, b)= & P\left\{U_{\delta}\left(T_{1}\right)<0\right\} \\
& =P\left\{u e^{\delta T_{1}}-Y_{1}<0\right\}=\int_{0}^{\infty} \bar{F}\left(u e^{\delta t}+c \bar{S}_{\bar{t} \mid}\right) d K(t) \\
h_{2}(u, a, b)= & P\left\{b \leq U_{\delta}\left(T_{1}\right) \leq a, U_{\delta}\left(T_{2}\right)<0\right\} \\
= & P\left\{b \leq u e^{\delta T_{1}}-Y_{1} \leq a, u e^{\delta T_{2}}-e^{\delta\left(T_{2}-T_{1}\right)} Y_{1}-Y_{2}<0\right\} \\
= & \int_{0}^{\infty} \int_{0}^{\infty} P\left\{b \leq u e^{\delta W_{1}}-Y_{1} \leq a, u e^{\delta\left(W_{1}+W_{2}\right)}\right. \\
& \left.-e^{\delta W_{2}} Y_{1}-Y_{2}<0 \mid Y_{1}=y, W_{1}=t\right\} d G(y, t) \\
= & \left.\int_{0}^{\infty} \int_{u e^{\delta t}-a}^{u\{} P\left(u e^{\delta t}-y\right) e^{\delta W_{2}}-Y_{2}<0\right\} d F\left(y+c \bar{S}_{\bar{t} \mid}\right) d K(t) \\
= & \int_{0}^{\infty} \int_{u e^{\delta t}+c \bar{S}_{\bar{t} \mid}-a}^{u \bar{S}_{\bar{t}}-b} P\left\{\left(u e^{\delta t}+c \bar{S}_{\bar{t} \mid}-y\right) e^{\delta W_{2}}-Y_{2}<0\right\} d F(y) d K(t) \\
= & \int_{0}^{\infty} \int_{u e^{\delta t}+c \bar{S}_{\bar{t} \mid}-b}^{u \bar{S}_{\bar{t} \mid}-a} h_{1}\left(u e^{\delta t}+c \bar{S}_{\bar{t} \mid}-y, a, b\right) d F(y) d K(t)
\end{aligned}
$$

By inductive assumption, when $n \geq 2$,

$$
\begin{aligned}
& h_{n}(u, a, b)=P\left\{b \leq U_{\delta}\left(T_{1}\right) \leq a,\right. \\
& \left., b \leq U_{\delta}\left(T_{2}\right) \leq a, \cdots, b \leq U_{\delta}\left(T_{n-1}\right) \leq a, U_{\delta}\left(T_{n}\right)<0\right\} \\
& =P\left\{b \leq u e^{\delta W_{1}}-Y_{1} \leq a, b \leq u e^{\delta\left(W_{1}+W_{2}\right)}-e^{\delta W_{2}} Y_{1}-Y_{2} \leq a, \cdots,\right. \\
& \left.\quad b \leq u e^{\delta \sum_{j=1}^{n-1} W_{j}}-\sum_{i=1}^{n-1} Y_{i} e^{\delta \sum_{j=i+1}^{n-1} W_{j}} \leq a, u e^{\delta \sum_{j=1}^{n} W_{j}}-\sum_{i=1}^{n} Y_{i} e^{\delta \sum_{j=i+1}^{n} W_{j}}<0\right\} \\
& =\int_{0}^{\infty} \int_{u e^{\delta t}-a}^{u e^{\delta t}-b} P\left\{b \leq\left(u e^{\delta t}-y\right) e^{\delta W_{2}}-Y_{2} \leq a,\right.
\end{aligned}
$$




$$
\begin{gathered}
\cdots, b \leq\left(u e^{\delta t}-y\right) e^{\delta \sum_{j=2}^{n-1} W_{j}}-\sum_{i=2}^{n-1} Y_{i} e^{\delta \sum_{j=i+1}^{n-1} W_{j}} \leq a, \\
\left.\left(u e^{\delta t}-y\right) e^{\delta \sum_{j=2}^{n} W_{j}}-\sum_{i=2}^{n} Y_{i} e^{\delta \sum_{j=i+1}^{n} W_{j}}<0\right\} d G(y, t) \\
=\int_{0}^{\infty} \int_{u e^{\delta t}-a}^{u e^{\delta t}-b} h_{n-1}\left(u e^{\delta t}-y, a, b\right) d F\left(y+c \bar{S}_{\bar{t} \mid}\right) d K(t) \\
=\int_{0}^{\infty} \int_{u e^{\delta t}+c \bar{S}_{\bar{T}}-b}^{u c \bar{S}_{\bar{t} \mid}-a} h_{n-1}\left(u e^{\delta t}+c \bar{S}_{\bar{t} \mid}-y, a, b\right) d F(y) d K(t)
\end{gathered}
$$

Theorem 2 when $b \leq u \leq a$, then $H(u, a, b)$ has the following integral equation

Proof

$$
\begin{gathered}
H(u, a, b)=\int_{0}^{\infty} \bar{F}\left(u e^{\delta t}+c \bar{S}_{\bar{t} \mid}\right) d K(t)+\int_{0}^{\infty} \int_{u e^{\delta t}+c \bar{S}_{\bar{t} \mid}-a}^{u t} H\left(u e^{\delta t}\right. \\
\left.+c \bar{S}_{\bar{t} \mid}-y, x\right) d F(y) d K(t)
\end{gathered}
$$

$$
\begin{aligned}
& H(u, a, b)=\sum_{n=1}^{\infty} h_{n}(u, a, b)=h_{1}(u, a, b)+\sum_{n=2}^{\infty} h_{n}(u, a, b) \\
& =h_{1}(u, a, b)+\sum_{n=2}^{\infty} \int_{0}^{\infty} \int_{u e^{\delta t}+c \bar{S}_{\bar{T} \mid}-a}^{u \bar{t}_{t}+c \overline{\bar{T}}_{\overline{\mid}}-b} h_{n-1}\left(u e^{\delta t}+c \bar{S}_{\bar{t} \mid}-y, a, b\right) d F(y) d K(t)
\end{aligned}
$$

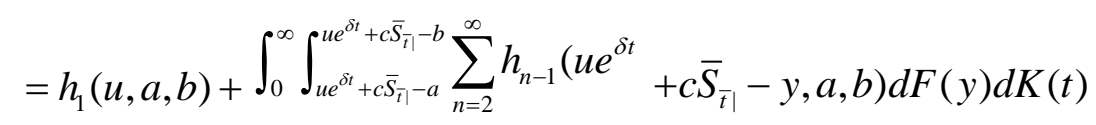

$$
\begin{aligned}
& =\int_{0}^{\infty} \bar{F}\left(u e^{\delta t}+c \bar{S}_{\bar{\tau} \mid}\right) d K(t)+\int_{0}^{\infty} \int_{u e^{\delta t}+c \bar{S}_{\bar{T} \mid} e^{\delta t}+a \bar{S}_{\bar{T}}-b}^{-b} H\left(u e^{\delta t}+c \bar{S}_{t}-y, a, b\right) d F(y) d K(t)
\end{aligned}
$$

Corollary 1 In Theorem 1 , let $b \rightarrow 0^{+}$, we can get the distribution function of the maximum surplus before the ruin $H(u, a)$

(1) when $a<u$, then $H(u, a)=0$

(2) when $a \geq u$, then $H(u, a)=\sum_{n=1}^{\infty} h_{n}(u, a)$, where $h_{1}(u, a)=\int_{0}^{\infty} \bar{F}\left(u e^{\delta t}+c \bar{S}_{\bar{t} \mid}\right) d K(t)$

When $n \geq 2$

\section{Proof}

$$
h_{n}(u, a)=\int_{0}^{\infty} \int_{u e^{\delta t}+c \bar{S}_{\bar{\tau} \mid} e^{\delta t}+a \bar{S}_{\overline{\mid}}} h_{n-1}\left(u e^{\delta t}+c \bar{S}_{\bar{t} \mid}-y, a\right) d F(y) d K(t)
$$

let

$$
b \rightarrow 0^{+}
$$

in Theorem 1, then we have Corollary 1.

Corollary 2 In Theorem 2 , let $b \rightarrow 0^{+}$, then $H(u, a)$ has the following integral equation

$$
\begin{aligned}
& H(u, a)=\int_{0}^{\infty} \bar{F}\left(u e^{\delta t}+c \bar{S}_{\bar{t} \mid}\right) d K(t)+\int_{0}^{\infty} \int_{u e^{\delta t}+c \bar{S}_{\bar{t} \mid}-a}^{\delta t}+\bar{c}_{\bar{t} \mid} H\left(u e^{\delta t}\right. \\
& \left.+c \bar{S}_{\bar{t} \mid}-y, a\right) d F(y) d K(t)
\end{aligned}
$$

\section{Proof}

let

$$
b \rightarrow 0^{+}
$$

in Theorem 2, then we have Corollary 2.

Corollary 3 In Theorem 1 , let $a \rightarrow+\infty$, we can get the distribution function of the minimum surplus before the ruin $H(u, b)$ 
(1) when $b>u$, then $H(u, b)=0$

(2) when $b \leq u$, then $H(u, b)=\sum_{n=1}^{\infty} h_{n}(u, b)$,

where

When $n \geq 2$

$$
h_{1}(u, b)=\int_{0}^{\infty} \bar{F}\left(u e^{\delta t}+c \bar{S}_{\bar{t} \mid}\right) d K(t)
$$

$$
h_{n}(u, b)=\int_{0}^{\infty} \int_{u e^{\delta t}+c \bar{S}_{\bar{\tau} \mid}}^{u e^{\delta t}+c \bar{S}_{\bar{T}}-b} h_{n-1}\left(u e^{\delta t}+c \bar{S}_{\bar{t} \mid}-y, b\right) d F(y) d K(t)
$$

Proof let $a \rightarrow+\infty$ in Theorem 1, then we have Corollary 3.

Corollary 4 In Theorem 2 , let $b \rightarrow 0^{+}$, then $H(u, a)$ has the following integral equation

$$
K(u, b)=\int_{0}^{\infty} \bar{F}\left(u e^{\delta t}+c \bar{S}_{\bar{t} \mid}\right) d K(t)+\int_{0}^{\infty} \int_{0}^{u e^{\delta t}+c \bar{S}_{\bar{t} \mid}-b} K\left(u e^{\delta t}+c \bar{S}_{\bar{t} \mid}-y, b\right) d F(y) d K(t)
$$

Proof let $a \rightarrow+\infty$ in Theorem 2, then we have Corollary 4 .

\section{Acknowledgment}

This work is supported by the Humanity and Social Science Youth Foundation of Ministry of Education of China (No. 15YJCZH055).

\section{References}

[1] B. Sundt, J. L. Tevgels. "Ruin estimates under interest force", Insurance: Mathematics and Economics, 16, p7-22, 1995.

[2] B. Sundt, J. L. Tevgels. "The adjustment function in ruin estimates under interest force", Insurance: Mathematics and Economics, 19, p85-94, 1997.

[3] H.L. Yang, L.H. Zhang. "On the distribution of surplus immediately after ruin under interest force”, Insurance: Mathematics and Economics, 29, p247-255, 2001(a).

[4] H.L. Yang, L.H. Zhang. "On the distribution of surplus immediately after ruin under interest force", Statistics and probability letters, 55, p329-338, 2001(b).

[5] H.L. Yang, L.H. Zhang. "The joint distribution of surplus immediately before ruin and the deficit at ruin under interestorce", North American Actuaria Journal, 5(3), p92-103, 2001c.

[6] R. Wu, Y.G. Du. "The renew risk model with constant interest force”, Journal of engineering mathematica, 19(1), p46-54, 2002.

[7] Q. M. Lin, R. M. Wang. "Calculation of ruin probabilities under a renew risk model with interest force", Journal of east china normal university, 1, p46-52, 2005.

[8] C. S. Zhang, R. Wu. "Union distribution of extreme value on classical risk", Acta mathematica scientia, 23(1), p25-30, 2003.

[9] H. Yang. "Non-exponential bounds for ruin probability with interest effect included", Scandinavian Actuarial Journal, 1, p66-79, 1998.

[10] N.L. Bowers, H.U. Gerber, J.C. Hickman, D.A. Jones, C.J. Nesbitt. Actuarial Mathematics, Society of Actuaries, Itasca, IL., 1986. 